\title{
Multi-objective Design of Transient Network Models
}

\author{
Bong Seog Jung, Misgana Muleta and Paul F. Boulos
}

\begin{abstract}
The optimal design of a water distribution system under transient conditions is formulated as a two-objective optimization problem. The objectives are minimization of the total pipe costs and maximization of the hydraulic reliability for the transient network design model. Unlike most optimization models in which demands are set to their end-of-life levels, this approach assumes that the demand loadings vary throughout the design life of the system. Evolutionary algorithms are applied to support efficient search for Pareto optimal solutions to the dual-objective optimization problem. An example application is presented and relevant conclusions are stated.
\end{abstract}

\section{INTRODUCTION}

A water distribution system is a complex network of pipes, pumps, valves, reservoirs, and storage tanks that is used to transport water from source to consumer. It is designed and operated to consistently deliver water in sufficient quantity, of acceptable quality, at appropriate pressure, as economically as possible. Traditionally, after choosing critical loading conditions in distribution system design, the greater of peak-hour demand or peak-day demand and a chosen fire flow, optimization methods are applied to select the most economical set of pipe sizes that will produce the desired range of pressures in the network. The rationale underlying the economical design is that, by selecting the smallest possible diameter set to minimize cost, pressures are marginally above an acceptable level for the specified design loading conditions. However, because the design problem is posed as a static one (i.e., the design loads are not treated as dynamic variables), the conventional design could well be suboptimal under transient pressures, or even seriously inadequate for other design events.

The optimization of a water distribution system under hydraulic transient conditions is difficult due to the complexity of the transient flow problem. Although a transient analysis is essential to estimate the worst-case events in the system, its inherent complexities have induced hydraulic design engineers to focus perhaps too much on steady state conditions. However, transient regimes in water distribution systems are both inevitable and naturally occurring (Boulos et al., 2005). Recent water quality studies have emphasized the need for transient analysis of large pipe 
networks to properly assess the potential level of intrusion associated with negative pressure events and the resulting impact on disinfectant residual effectiveness (Besner, 2007; Boulos et al., 2006; NRC, 2006; Fleming et al., 2006; Friedman et al., 2004; LeChevallier et al., 2002-2003; Kirmeyer et al., 2001). In reality, since all pipeline systems leak and hydraulic transients will occur continuously in a water distribution system, it is not surprising that low-pressure transient events offer considerable potential to draw untreated and possibly hazardous water into the piping system.

Numerous hydraulic transient approaches have been developed to identify system weak points, to predict the potentially destructive effects of hydraulic transients under various worst-case scenarios, and to evaluate how they may possibly be eliminated or controlled (e.g., Wylie and Streeter, 1993; Chaudhry, 1987; Thorley, 2004; Boulos et al., 2005; Wood et al., 2005; Ghidaoui et al., 2005; Jung et al., 2007). In particular, Boulos et al. (2005) provided a detailed transient analysis flow chart for the selection of components for surge control and suppression in water distribution systems, and concluded that a transient analysis should always be carried out to determine the impact of each proposed strategy on the resulting system performance. Jung et al. (2007) argued that only a systematic transient analysis can be expected to resolve complex transient characterizations and adequately protect water distribution systems.

In addition, optimization methods have been widely applied to many problems associated with water distribution system design, management and operation. Alperovits and Shamir (1977) applied linear programming and Lansey and Mays (1989) suggested using nonlinear programming to optimize component sizing and the operational decisions arising in water distribution systems. Simpson et al. (1994) and Dandy et al. (1996) compared a Genetic Algorithm (GA) approach to both complete enumeration and nonlinear programming in the context of pipeline optimization. Much of the pipeline optimization literature has been concerned with systems under steady or near steady flow conditions; however, a few optimization approaches dealt with operating conditions pertaining to system integrity, safety and performance. Laine and Karney (1997) applied optimization to a simple pipeline connecting a pump and a storage reservoir. A complete enumeration scheme as well as a probabilistic selection procedure were incorporated with both transient and steady state analysis. Lingireddy et al. (2000) described a surge tank design model based on a bi-level genetic optimization framework that produces optimal tank sizes while satisfying a specified set of pressure constraints. Jung and Karney (2004) considered the impact of transients on the choice of optimal diameter in a network considering both steady and transient criteria. More recently, Jung and Karney (2006) presented an optimum selection approach of hydraulic devices for water hammer control in a water distribution system. GA and Particle Swarm Optimization (PSO) were used to optimize the preliminary selection, sizing and placement of surge protection devices.

In this paper, the optimal design of a water distribution system under transient conditions is formulated as a two-objective optimization problem. The objectives are 
minimization of the total pipe costs and maximization of the hydraulic reliability for the transient network design model. Unlike most optimization models in which demands are set to their end-of-life levels, this approach assumes that the demand loadings vary throughout the design life of the system. Evolutionary algorithms are applied to support efficient search for Pareto optimal solutions to the dual-objective (pipe costs and hydraulic reliability) optimization problem. The model is tested (by simulation) on the classical New York tunnel system and relevant conclusions are stated.

\section{MATHEMATICAL FORMULATION}

Whether designing a water distribution system (WDS) using trial-and-error enumeration methods or with formal optimization tools, a broad range of concerns must be considered. Cost is likely to be the primary emphasis and includes the costs for construction, operation and maintenance. The initial capital investment for the system includes pipes, pumps, tanks, and valves. Energy consumption occurs over time as the system is operated. The main constraints are that the nodal demands are supplied at a minimum pressure. In addition, the network flows and pressure heads must satisfy the governing equilibrium laws of conservation of energy and mass.

In this paper, the optimal design of a water distribution system under transient conditions is formulated as a two-objective optimization problem. The first objective is formulated in Equation (1) as a least-cost optimization problem with the selection of pipe diameters as the decision variables. The second objective is to maximize hydraulic reliability for the transient network design model. This objective given in Equation (2) is formulated to minimize the integration of the transient pressures that are lower than the minimum required level (e.g., datum) or higher than the maximum allowable transient pressure level (e.g., pipe ratings). In contract with traditional optimization models in which demands are set to their end-of-life levels, this approach assumes that the demand loadings vary throughout the design life of the system. The pipe network layout, nodal demands, and minimum head requirements are assumed known. The optimal design of water distribution networks can be stated mathematically as:

$$
\begin{aligned}
& \operatorname{minimize} \sum_{k \in N_{\text {pipe }}} C_{k}\left(D_{k}, L_{k}\right) \\
& \text { minimize } \sum_{i \in N_{\text {node }}} \int^{\forall T i m e}\left|H_{i}(t)\right| d t, \text { where } H<H_{\text {min }}^{*} \text { or } H>H_{\text {max }}^{*}
\end{aligned}
$$

Subject to the governing transient equations

$$
\frac{1}{g A_{p}} \frac{\partial Q}{\partial t}+\frac{\partial H}{\partial x}+\frac{R}{\Delta x} Q|Q|^{n-1}=0
$$




$$
\frac{\partial H}{\partial t}+\frac{a^{2}}{g A_{p}} \frac{\partial Q}{\partial x}=0
$$

and a set of algebraic constraints:

$$
\begin{aligned}
& H_{i}(t)=C_{1}, Q_{i}(t)=C_{2}, \text { where } t=0, \forall i \in N_{\text {node }} \\
& f\left(H_{i}(t), Q_{i}(t)\right)=C_{3} \text {, where } t>0, i=\text { boundary nodes } \\
& H_{i}(t) \geq H_{\min i} \text {, where } t=0, \forall i \in N_{\text {node }} \\
& D_{k} \in\{D\}, \quad \forall k \in N_{\text {pipe }}
\end{aligned}
$$

where $D_{k}=$ discrete pipe diameters selected from the set of commercially available pipe sizes $\{D\}$ [Equation (8)]; $C_{k}\left(D_{k} L_{k}\right)=$ cost of pipe $k$ with diameter $D_{k}$ and the length $L_{k} ; H=$ piezometric head; and $H_{\text {max }}^{*}$ and $H_{\text {min }}^{*}$ are the maximum and minimum permissible heads (say representing pipe ratings or health concerns for negative pressures), respectively. Equations (3) and (4) represent the momentum equation and mass conservation for transient flow in closed conduits (Wylie and Streeter, 1993). Here, $x$ is distance long the centerline of the conduit; $t$ is time; $Q=$ volumetric flow rate; $a=$ celerity of the shock wave; $A_{p}=$ cross-sectional area of the pipe; and $g=$ acceleration due to gravity. The friction term $R$ in the Equation (3) can be represented by

Darcy-Weisbach: $R=f_{p} x / 2 g D_{p} A_{p}^{2}, n=2$

or

Hazen Williams: $R=x /\left(0.278 C D_{p}^{2.63}\right)^{1 / 0.54}, n=1 / 0.54$

in which $f_{p}=$ Darcy-Weisbach friction factor; and $C=$ Hazen-Williams roughness coefficient. The two hyperbolic partial differential equations in (3) and (4) are subject to the initial conditions of Equation (5) and boundary conditions of Equation (6), where $C_{1}, C_{2}$ and $C_{3}$ are constants. Initial conditions are typically taken as steady. Simple boundary conditions of constant reservoir level and fixed demand are assumed, but combined relationships between $H$ and $Q$ are typical for most boundaries. Equation (7) requires that the nodal pressure $H$ for any node $i$ (where total number of nodes is $N_{\text {node }}$ ) is equal to or greater than a specified minimum pressure $H_{\min }$ for steady state condition.

The remaining and challenging question is how to apply an optimization method to the suggested problem of water distribution optimization. Gradient-based mathematical optimization methods (Alperovits and Shamir, 1977; Bhave, 1985) have been widely applied and have provided efficient computational procedures for 
achieving a lower cost solution but the methods suffered from some disadvantages, such as: (1) being ineffective at reaching the least cost solution due to zero-gradient optimality criteria, which easily trapped a search process at a local optimal solution; (2) the lack of flexibility in handling discrete design variables and optimizing a partial network that is often required for many practical engineering designs; (3) the complexity of implementing and using the techniques (Wu and Simpson, 2001); and (4) the requirement to compute either first or second derivative information in order to generate improvements in the objective function. Recently, several researchers have used genetic algorithm (GA) optimization for solving such complex WDS optimization (Simpson et al., 1994; Dandy et al., 1996). Ant Colony Optimization (Maier et al., 2003) and Shuffled Frog Leaping Algorithm (Eusuff and Lansey, 2003) have also been applied for obtaining specific optimal designs of WDSs. These methods offers significant advantages over gradient-based optimization approaches in that they do not require any gradient information and search for the optimal solution by continuing to evaluate multiple solution vectors simultaneously.

For the given dual-objective (pipe costs and hydraulic reliability for the transient network design model) problem, genetic optimization is used to circumvent subjective decision making and to generate Pareto optimal solutions for the multiobjective optimization problem. In this study, Nondominated Sorting GA (NSGA), developed by Srinivas and Deb (1994), is employed to find the Pareto optimal solutions. Figure 1 depicts a flowchart of the framework for optimizing the pipeline system considering the dual-objective problem. First, an optimization program initializes the pipe sizes as decision variables, and the pipe cost is calculated. The hydraulic model then analyzes the given system and uses the optimization program to check if the solution satisfies the required constraints given by Equations (3) to (8) and then computes the second objective function shown in Equation (2). With the dual objective function values, the optimization model then evaluates the system and creates a new set of system alternatives for the next iteration. The iterations continue until the optimal solution is reached. This allows rapid solutions to be obtained with a minimal computational overhead.

Decision variable: Pipe size

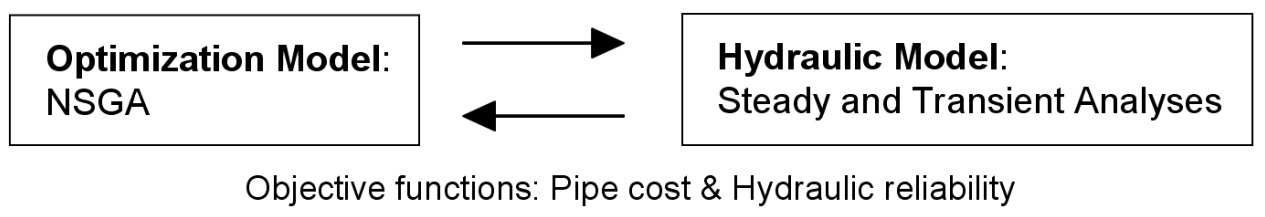

Figure 1. Flowchart of pipeline optimization

\section{CASE STUDY}

The proposed method is illustrated using the classical New York tunnel system (Schaake and Lai, 1969). The network, which is shown in Figure 2, has been extensively studied for steady state conditions. It comprises 22 nodes (20 demand 
nodes), 21 pipes, and one source node. The system is gravity driven and draws water from the Hillview reservoir to the downstream network. The objective of the optimization problem is to add new pipes parallel to the existing ones. The new pipe diameters need to be selected from 15 available sizes. A single demand pattern $(57,130 \mathrm{~L} / \mathrm{s})$ was considered and a minimum allowable hydraulic grade was specified for each node. The network and cost data are given in Dandy et al. (1996).

Since the system was first examined in 1969 by Schaake and Lai, numerous subsequent researchers have used it to test the numerical effectiveness, efficacy and performance of their respective techniques (Dandy et al., 1996; Savic and Walters, 1997; Wu et al., 2001; Eusuff and Lansey, 2003; Maier et al., 2003). However, all these approaches were based on steady state optimization only. In this paper, not only the steady state problem is considered, but also the transient analysis is included in the optimization process. By doing so, it is shown that different design decisions would be required, and the restrictive search based on limited operating conditions is likely suboptimal for a broader range of demand loadings.

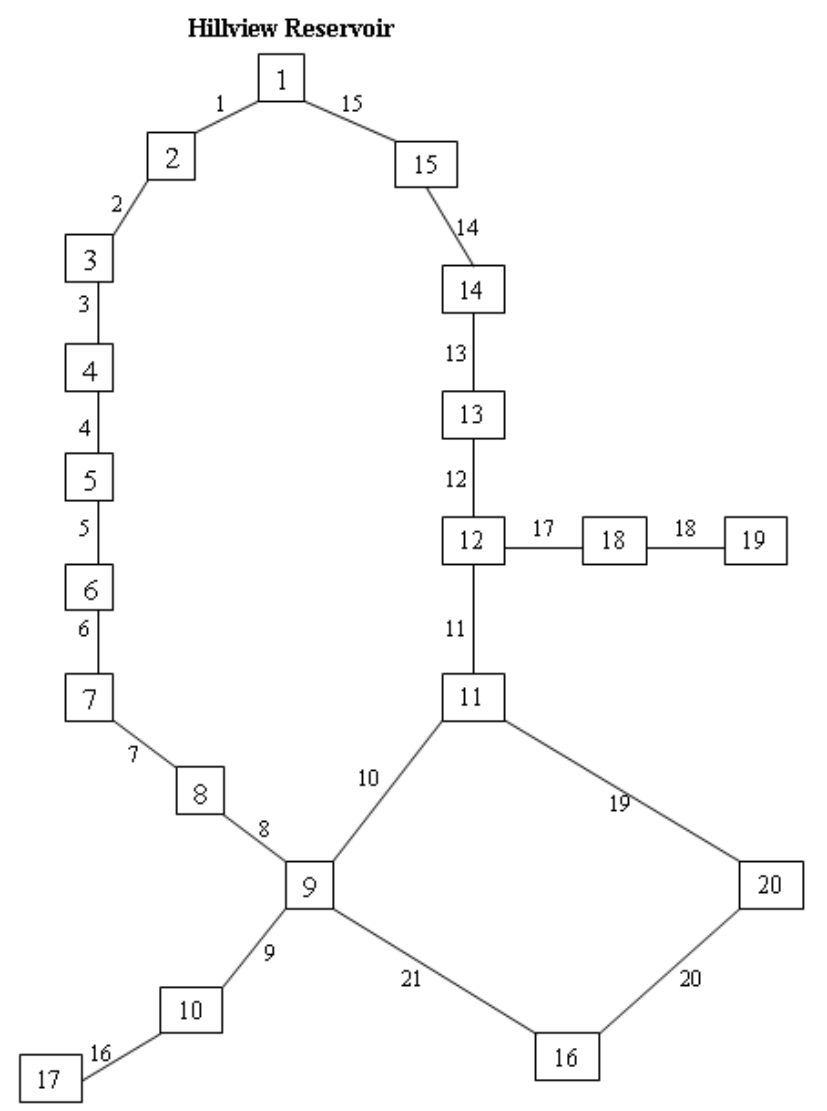

Figure 2. New York tunnel system schematic

To introduce transient conditions into this case study, a variety of possible causes could be selected. For convenience, a valve opening that increases the demand at node $10 \mathrm{from} 28 \mathrm{~L} / \mathrm{s}$ to $4814 \mathrm{~L} / \mathrm{s}$ for a 1 second period is chosen to characterize the transient performance of the system. This increased demand may represent a fire 
flow, a pipe burst, an operator error or a temporary increased in water consumption. The maximum permissible heads $H_{\text {max }}^{*}$ and $H_{\text {min }}^{*}$ in Equation (2) are assumed to be $304.8 \mathrm{~m}$ and $54.9 \mathrm{~m}$, respectively, for the whole system. Due to the rapid demand increase at node 10 , a reduced pressure wave moves through the system. This wave is reflected from the upstream reservoir and then propagates back and forth in the system, being tracked numerically using the method of characteristics (Wylie and Streeter, 1993). The different pipe lengths and sizes in the system create uneven computational lengths in the characteristic grids. The smallest Courant number in the system is 0.019 and it can be adjusted to unity by dividing the pipes into smaller computational units. The process of discretization is repeated until the smallest Courant number exceeds 0.75. After discretization, the smallest Courant number and the computational time step are 0.755 and $1.11 \mathrm{~s}$, respectively. For the uneven computational units, a linear timeline interpolation is used to obtain head and flow at a grid point in the characteristic mesh.

The multi-objective method, NSGA, is considered to satisfy Equations (1) to (8). For this problem, the probability of mutation is set to 0.025 , the probability of (single-point) crossover is set to 0.9 , the population size is set to 400 , the length of each chromosome is set to 84 , and the simulations are run for 100 generations. For this problem, 16 decision variables including the "do nothing" option make up a solution space of $16^{21}$ or $1.93 \times 10^{25}$ possible pipe combinations. The NSGA initializes the population of pipe diameters, and then calculates the cost of pipelines and the hydraulic reliability of transient network design model satisfying the given constraints, and then create a new population for the next generation. After 100 generations, the resulting Pareto optimal solutions are shown in Figure 3 and Table 1. The hydraulic reliability results indicate that the previous approaches that considered only steady state design are inadequate for coping with water hammer events. The results also suggest that the proper sizing of pipe diameters is crucially important to prevent water hammer as well as to decrease cost.

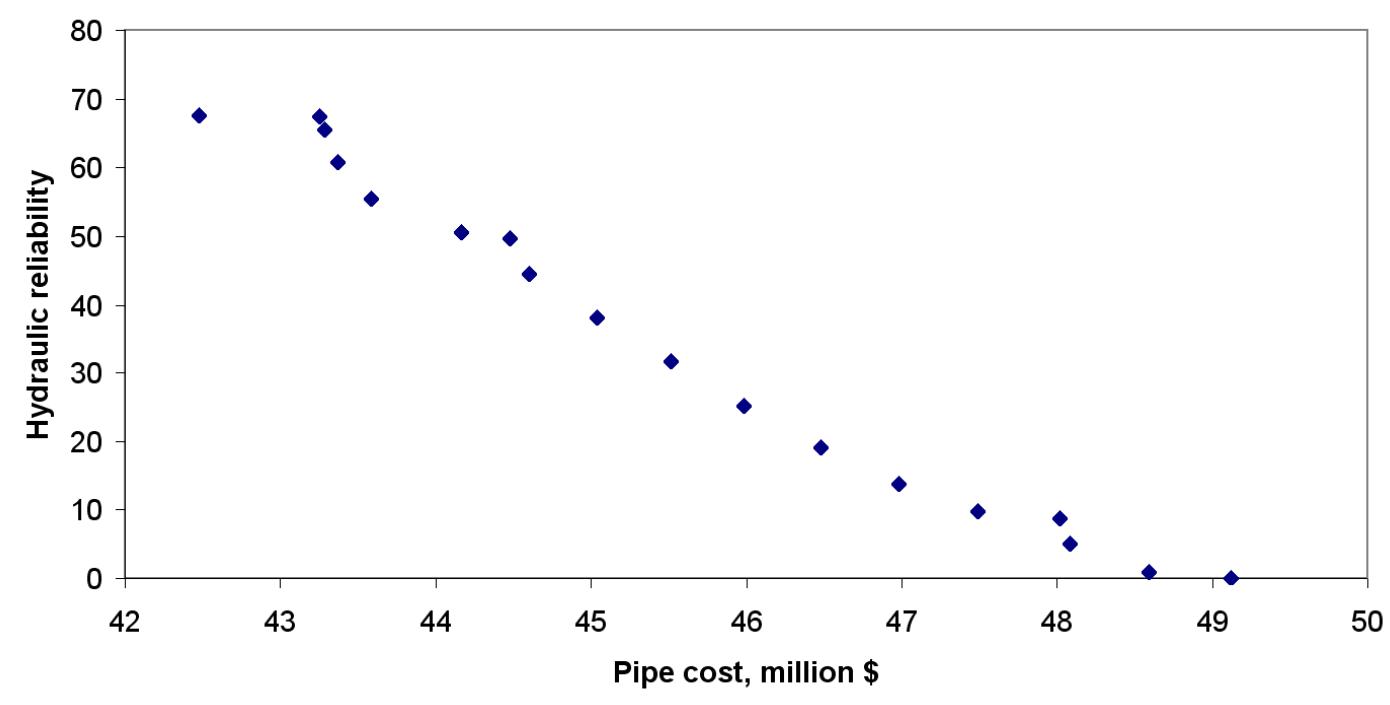

Figure 3. Pareto optimal solutions of pipe cost and hydraulic reliability 
Table 1. Pareto optimal solutions for pipe sizes

\begin{tabular}{cccc}
\hline Pipe & \multicolumn{3}{c}{ Pipe size $(\mathrm{mm})$} \\
\hline $1-8$ & - & - & - \\
9 & 3900 & 2700 & - \\
$11-15$ & - & - & - \\
16 & 3000 & 3000 & 3000 \\
17 & 2100 & 2100 & 2100 \\
18 & 3000 & 3000 & 3000 \\
19 & 2100 & 1800 & 1800 \\
20 & 3000 & 3000 & 3000 \\
21 & - & - & - \\
22 & 1500 & 1500 & 1500 \\
\hline Pipe Cost, \$ million & 49.12108 & 45.98196 & 42.47795 \\
\hline Hydraulic Reliability & 0 & 25.21 & 67.51 \\
\hline
\end{tabular}

\section{CONCLUSION}

Transient analysis, despite its significant concern for water distribution system design, is a complicated problem and so is the optimization of a transient control strategy for water distribution systems. The purpose of this paper is to obtain the optimal design of a pipe network by considering simultaneously steady and transient states. The objectives are minimization of the total pipe costs and maximization of the hydraulic reliability for the transient network design model. Unlike most optimization models in which demands are set to their end-of-life levels, this approach assumes that the demand loadings vary throughout the design life of the system. To achieve the dual-objectives, evolutionary algorithms based multi-objective optimization is used to circumvent subjective decision making and generate Pareto optimal solutions.

The case study using the New York tunnel system indicates that the previous approaches that considered steady state design only are inadequate for coping with water hammer events. In addition, the study suggests that proper sizing of pipe diameters is crucially important to prevent water hammer as well as to decrease cost. Although this paper considers the optimal selection of pipe diameters for a surge protection strategy, a more global and comprehensive approach is ultimately needed. Water distribution system optimization should also consider, in addition to pipe size, the transient properties (e.g., operation speed), system characteristics (e.g., system topography, pipe material and thickness) and transient protection devices. This comprehensive design framework will offer a more complete range of systematic surge protection strategies resulting in more reliable cost optimization solutions.

\section{REFERENCES}

Alperovits, E., and Shamir, U. (1977). "Design of optimal water distribution systems." Water Resources Research, 13(6), 885-900. 
Besner, M. C. (2007). Risk evaluation of drinking water distribution system contamination due to operation and maintenance activities. Ph.D Dissertation, Universite of Montreal, Quebec, Canada.

Bhave, P. R. (1985). "Optimal expansion of water distribution systems." Journal of Environmental Engineering, ASCE, 111(2), 177-197.

Boulos, P. F., Karney, B. W., Wood, D. J., and Lingireddy, S. (2005). "Hydraulic transient guidelines for protecting water distribution systems." Journal of the American Water Works Association, 97(5), 111-124.

Boulos, P. F., Lansey, K. E., and Karney, B. W. (2006). Comprehensive water distribution systems analysis handbook for engineers and planners. $2^{\text {nd }}$ Edition, MWH Soft, Inc. Publ., Pasadena, CA.

Chaudhry, M. H. (1987). Applied hydraulic transients. 2d ed., Van Nostrand Reinhold, NY.

Dandy, G. C., Simpson, A. R., and Murphy, L. J. (1996). "An improved genetic algorithm for pipe network optimization." Water Resources Research, 32(2), 449-458.

Eusuff, M. M., and Lansey, K. E. (2003). "Optimization of water distribution network design using the shuffled frog leaping algorithm." Journal of Water Resources Planning and Management, ASCE, 129(3), 210-225.

Fleming, K. K., Gullick, R. W., Dugandzic, J. P., and LeChevallier, M. W. (2006). Susceptibility of distribution systems to negative pressure transients. AWWARF, Denver, CO.

Friedman, M., Radder, L., Harrison, S., Howie, D., Britton, M., Boyd, G., Wang, H., Gullick, R., LeChevallier, M., Wood, D, and Funk, J. (2004). Verification and control of pressure transients and intrusion in distribution systems. AWWARF, Denver, CO.

Ghidaoui, M. S., Zhao, M., McInnis, D. A., and Axworthy, D. H. (2005). "A Review of water hammer theory and practice." Applied Mechanics Reviews, 58, 49-76.

Jung, B. S., and Karney, B. W. (2004). "Fluid transients and pipeline optimization using GA and PSO: the diameter connection." Urban Water Journal, 1(2), 167-176.

Jung, B. S., and Karney, B. W. (2006). "Optimization of transient protection devices using GA and PSO approaches." Journal of Water Resources Planning and Management, ASCE, 132(1), 44-52.

Jung, B. S., Karney, B. W., Boulos, P. F., and Wood, D. J. (2007). "The Need for comprehensive transient analysis of distribution systems." Journal of the American Water Works Association, 99(1), 112-123.

Kirmeyer, G. J., Friedman, M., Martel, K., Howie, D., LeChevallier, M., Abbaszadegan, M., Karim, M., Funk, J., and Harbour, J. (2001). Pathogen intrusion into the distribution system. AWWARF, Denver, CO.

Laine, D. A., and Karney, B. W. (1997). "Transient analysis and optimization in pipeline - a numerical exploration." 3rd International Conference on Water Pipeline Systems, Hague, Netherlands, 281-296.

Lansey, K. E., and Mays, L. W. (1989). "Optimization model for water distribution system design." Journal of Hydraulic Engineering, ASCE, 115(10), 14011418 . 
LeChevallier, M. W., Gullick, R. W., and Karim, M. R. (2002). The potential for health risks from intrusion of contaminants into the distribution system from pressure transients. American Water Works Service Company, Inc., Voorhees, NJ: Distribution System White Paper prepared for the USEPA (http://www.epa.gov/safewater/tcr/pdf/intrusion.pdf).

LeChevallier, M. W., Gullick, R. W., Karim, M. R., Friedman, M., and Funk, J. E. (2003). "The potential for health risks from intrusion of contaminants into the distribution system from pressure transients." Journal of Water and Health, 1(1), 3-14.

Lingireddy, S., Funk, J. E., and Wang, H. (2000). "Genetic algorithms in optimizing transient suppression devices." Proc. ASCE 2000 Joint Conference on Water Resources Engineering and Water Resources Planning and Management, Minneapolis, MN.

Maier, H. R., Simpson, A. R., Zecchin, A. C., Foong, W. K., Phang, K. Y., Seah, H. Y., and Tan, C. L. (2003). "Ant colony optimization for design of water distribution systems." Journal of Water Resources Planning and Management, ASCE, 129(3), 200-209.

NRC (National Research Council/National Academies) (2006). Drinking water distribution systems: assessing and reducing risks. The National Academies Press, Washington DC.

Savic, D. A., and Walters, G. A. (1997). "Genetic algorithms for least-cost design of water distribution network." Journal of Water Resources Planning and Management, ASCE, 123(2), 67-77.

Schaake, J., and Lai, D. (1969). "Linear programming and dynamic programming applications to water distribution network design." Rep. 116, Dept. of Civ. Engrg., Massachusetts Inst. of Technol., Cambridge, MA.

Simpson, A. R., Dandy, G. C., and Murphy, L. J. (1994). "Genetic algorithms compared to other techniques for pipe optimization." Journal of Water Resources Planning and Management, ASCE, 120(4), 423-443.

Srinivas, N., and Deb, K. (1994). "Multi-objective function optimization using nondominated sorting genetic algorithms." Evol. Comput., 2(3), 221-248.

Thorley, A. R. D. (2004). Fluid transients in pipeline systems, $2^{\text {nd }}$ edition. D\&L George Ltd., Herts, England.

Wood, D. J., Lingireddy, S., Boulos, P. F., Karney, B. W., and McPherson, D. L. (2005). "Numerical methods for modeling transient flow in distribution systems." Journal of the American Water Works Association, 97(7), 104-115.

Wu, Z. Y., and Simpson, A. R. (2001). "Competent genetic-evolutionary optimization of water distribution systems." Journal of Computing in Civil Engineering, ASCE, 15(2), 89-101.

Wu, Z. Y., Boulos, P. F., Orr, C. H., and Ro, J. J. (2001). "Using genetic algorithms to rehabilitate water distribution systems." Journal of the American Water Works Association, 93(11), 74-85.

Wylie, E. B., and Streeter, V. L. (1993). Fluid transients in systems. Prentice Hall, Englewood Cliffs, NJ. 